\title{
Robust Heart Sound Segmentation and Detection using biased Cramer-Rao Lower Bound Estimation and Variance Algorithm
}

\author{
D. Sandeep Vara Sankar, R. Aditya, S. Gopi Krishna, P. Atchuta Rao
}

\begin{abstract}
Cardiac disorders are the most common infirmity suffered by the human race. Medical research states that worldwide almost $30 \%$ of the deaths are caused because of cardiovascular diseases. Even though many procedures such as Echocardiography, Carotid pulse and Electrocardiography are available, the conventional approach followed for detecting abnormalities are carried by hearing to the heart rhythms. This proposed study enables the health care physicians to diagnose pathologies of highly non-stationary heart sounds efficiently by using the second-order statistics of the signal. In this paper, a preprocessing methodology is used for removing noise caused by the lower frequency components and to focus only on the primary components $S 1 \& S 2$, which are further feature extracted by using principal component analysis (PCA). A proposed variance algorithm (VA) is developed to identify the boundary locations of heart sounds and segment the featured signals into series of cardiac cycles. Further, we developed a modified variance algorithm (MVA) using biased Cramer-Rao lower bound (CRLB) to estimate the heart sound $(H S)$ signals that exhibit minimum variance and extract finer boundary locations of the signal components which helps in identifying $S 1 \& S 2$ positions. When compared with PCA, VA and Shannon energy (SE) methods for the same dataset, the MVA scheme exhibits very low normalized mean square error (NMSE) in extracting boundary locations by $-71.60 \pm 0.25 d B$ and achieves high cardiac cycle segmentation ratio of $97.78 \pm 0.98 \%$. A brief analysis of the results showed that the proposed MVA scheme using biased CRLB exhibits $97.4 \pm 1.2 \%$ accuracy in identifying $S 1$ and $S 2$ heart sounds.
\end{abstract}

Keywords : Principal Component Analysis, Segmentation, Minimum Variance Unbiased Estimator, Cramer-Rao Lower Bound, Mean Square Error, Phonocardiogram.

\section{INTRODUCTION}

$\mathrm{T}$ he heart is an important and powerful muscle that pumps blood through the circulatory system and the sounds generated are due to turbulent flow of blood through open and close mechanism of heart valves. Listening to the heart sounds is one of the preliminary tool for diagnosing cardiac ailments. Literature states that the physicians have very poor auscultation skills in determining the condition of ailments. Although techniques like ECG, MRI, and echocardiogram exist, they are unavailable to the remote segments of the

Revised Manuscript Received on December 13, 2019.

Diddi Sandeep Vara Sankar*, Department ofEECE, GIT, GITAM (Deemed to be University), India. Email: sandeep.diddi@gitam.edu

Rallapti Aditya, Department of EECE, GIT, GITAM (Deemed to be University), India. Email: aditya.rallapati@gitam.edu

Sammeta Gopi krishnaDepartment of EECE, GIT, GITAM (Deemed to be University), India. Email: gopikrishna.sammeta@ gitam.edu

Pudu Atchuta RaoDepartment of EECE, GIT, GITAM (Deemed to be University), India. Email: atchutarao.pudu@gitam.edu

community and quite costly. Even though monitoring of HSs through a stethoscope is a fundamental tool for diagnosing heart diseases, it requires a lot of experience and practice. Thus auscultation mechanism needs to be improved for primary screening purposes [2].

Murmurs are due to the dysfunction in the heart valves which causes additional sounds along with the primary sounds. The additional sounds may be pathological and are caused due to narrowed valves or regurgitant flow through septal defects or valves [15]. A normal phonocardiogram (PCG) signal consists of two regular and repetitive thuds called primary heart sounds (S1 and S2). In addition to these, two weak sounds $\mathrm{S} 3$ and $\mathrm{S} 4$ are produced during the diastolic phase of the cardiac cycle. Researches show that each pathology has a unique murmur pattern that resembles the necessity for diagnosing these ailments and developing a model for clinical purposes.

The highly non-stationarity nature of the heart sound (HS) signal cannot be analyzed by a simple frequency transform. So a mechanism in time-frequency planeto understand the non-stationary characteristics of heart sounds is provided using short Time Fourier transform - STFT by AbdelghaniDjebbari et al. [3]. The fixed window length in STFT provides a trade-off between time and frequency resolutions which can beeliminated by using wavelets. S.M Lee et al. [4] described that wavelet transform is helpful in the feature extraction of HS signals by comparing it with STFT. H. Liang et al. [5] uses Shannon energy to extract the envelope of the HS signal energy to understand the structure and characteristics of HS. The method provides very poor results in cases where the signals are dominated by murmurs. Time-frequency analysis is extensively used for the analysis of HS signals and segmentation of pathological HS signal using this approach is proposed by D. Boutana et al. [6] which also helps to extractimportant information from features for diagnosing pathologies in the systolic phase Time-frequency analysis suffers from multiplicative noises, probabilistic models require prior information of the heart sound signals which is very difficult and also not reliable. Even though model based approaches are sensitive to modeling atechnique of homomorphic filtering based on the self-organizing probabilistic model can be used to detect and identify primary heart sounds. The scalable smoothing property and itscapability in handling the splittingproblemand notched peaks help homomorphic filtering as a choice for segmentation. 
Wavelet analysis is the most widely used approach to analyze PCG signals by extracting features, for segmentation and classification. Christer Ahlstrom et al. [7] proposed a method to classify systolic murmursby using wavelet feature extraction. The method detects murmur and HS components separately. M.A.R Santos et al. [8] demonstrated that Daubechies (4-19), Morlet and Meyer wavelets have the property of orthogonality and can be used for analyzing and extracting features from PCG signals. In [12] Chrysa D. P et al. proposed an ensemble empirical mode decomposition using kurtosis features to segment and extract the primary components (S1 and S2). A fuzzy-based joint cycle time-frequency domain is proposed by Hong Tang et al. [10] to detect primary components from noisy HS in which HS signals are decomposed into atoms characterizing time delay, frequency, amplitude and phase using $\mathrm{R}$ peak to determine the beginning of a cardiac cycle. Compressing murmurs [13] from the pathological HS using adaptive thresholding has a significance in identifying primary components where wavelets are used as features to segment normal and murmur portions depending on their estimates. Optimal estimators using biased Cramer-Rao lower bound (CRLB) proposed in [14], shows that for all the bias gradient estimators, penalized maximum likelihood estimator achieves uniform CRLB and it can be used for parametric estimations of superimposed signals [22].

\section{MATERIAL}

The biomedical records are sourced from [23] and [24] which are sampled at $22050 \mathrm{~Hz}$ sampling frequency with 16-bit accuracy. The records consist of both men and women with moderate and severe heart valve diseases, and normal condition whose characteristics are summarized in Table 1. Acquisitions made in the database have HS signals starting with either S1 or S2. The pathological record consists of different valve diseases like pulmonic, tricuspid, aortic, and mitral valve diseases. The data were recorded from different auscultation positions and refined to constant time of 15 seconds each.

Table I. Details of Experimental Testing Dataset

\begin{tabular}{|c|c|c|c|c|}
\hline \multicolumn{2}{|l|}{$\begin{array}{l}\text { Category } \\
\text { No (Gender) - (Severity) }\end{array}$} & \multicolumn{2}{|c|}{$\begin{array}{c}\text { Subjects } \\
\text { Count }\end{array}$} & Zardiac Cycle \\
\hline Normal & 30 & $(16 \mathrm{M} / 14 \mathrm{~F}$ & & 1535 \\
\hline Aortic Stenosis (AS) & 11 & $(7 \mathrm{M} / 4 \mathrm{~F})$ & $-(5 \mathrm{Mo}, 6 \mathrm{~S})$ & 652 \\
\hline $\begin{array}{l}\text { Pulmonic Stenosis } \\
\text { (PS) }\end{array}$ & 7 & $(4 \mathrm{M} / 3 \mathrm{~F})$ & - $(3 \mathrm{Mo}, 4 \mathrm{~S})$ & 445 \\
\hline $\begin{array}{l}\text { Aortic Regurgitation } \\
\text { (AR) }\end{array}$ & 6 & $(4 \mathrm{M} / 2 \mathrm{~F})$ & - (1Mo,5S) & 348 \\
\hline Mitral Stenosis (MS) & 13 & $(7 \mathrm{M} / 6 \mathrm{~F})$ & - $(10 \mathrm{Mo}, 3 \mathrm{~S})$ & 782 \\
\hline $\begin{array}{l}\text { Mitral Regurgitation } \\
\text { (MR) }\end{array}$ & 9 & $(5 \mathrm{M} / 4 \mathrm{~F})$ & - $(5 \mathrm{Mo}, 4 \mathrm{~S})$ & 486 \\
\hline $\begin{array}{c}\text { Tricuspid Stenosis } \\
\text { (TS) }\end{array}$ & 10 & $(7 \mathrm{M} / 3 \mathrm{~F})$ & $-(5 \mathrm{Mo}, 5 \mathrm{~S})$ & 498 \\
\hline Total & $\begin{array}{l}86 \\
-(29\end{array}$ & $\begin{array}{l}(50 \mathrm{M} / 36 \mathrm{~F} \\
9 \mathrm{Mo}, 27 \mathrm{~S},\end{array}$ & $0 \mathrm{~N})$ & 4746 \\
\hline
\end{tabular}

Mo - Moderate, S - Severe, N - Normal, M - Male, F - Female

\section{RESULT \& PROCEDURE FOR SEGMENTING PRIMARY COMPONENTS}

\section{A. Preprocessing}

Noise interference plays an important role during the acquisition process [15]. These noises aremay be due to respiratory tracts or auscultation positions or environmental turbulences or even measuring equipment that is used. The frequency range of these kinds of disturbances lies approximatelybelow $40 \mathrm{~Hz}$ which has to be removed at theearly stagesfor accurateboundary detection. So a filtering technique using a high pass filter is performed to eliminate all the noises below $40 \mathrm{~Hz}$. Researchers have demonstrated that the frequency range of primary HSs due to blood flow in the heart valves are in between 40 to $200 \mathrm{~Hz}$ and that of murmurs due to heart abnormalities are up to $600 \mathrm{~Hz}$ [20].A general block diagram for segmenting heart sounds from the PCG signal is shown in Fig. 1 andthe frequency distribution of the PCG signal for AS is shown in Fig. 2. The result makes it clear that it is unnecessary to consider all the frequency components for analysis and diagnosis purposes. So the HS signals are decimated by a factor of 12 to remove all the insignificant frequencies. The decimation process is conducted in both forward and reverse direction, so that phase distortion can be removed.

\section{B. Feature Extraction}

Feature extraction reduces the redundancy by transforming the input signal into the feature set which carries entire information of the input data. HS signals are highly transient and require the best techniques to extract features, while wavelets have the capability of handling these transients by using their scaling and translational property [17]. Although wavelets play a significant role in understanding the nature of the coefficients, choosing the best wavelet that has the capability of extracting primary components is very important. [8] Confirms that Meyer and Daubechies wavelets are the good constructs for the analysis of HS signals. So Daubechies-15 (db-15) wavelet is chosen as the mother wavelet for decomposing the HS signal. The decomposition is computed up to 5 levels and corresponding wavelet coefficients are calculated. The decomposed wavelets are further synthesized to attain a set of features at optimum temporal resolution. Although feature sets are obtained, selecting the best feature which resembles primary components is still a tedious task that cannot be possible with wavelets. So a process that picks the selective feature that propagates primary components is required, which makes it possible by using principal component analysis (PCA) [19]. In PCA, each Eigenvalue measures the amount of information captured in the direction of the corresponding Eigenvector. PCA transforms HS signal into an ordered set of transformed features where each principal component (PC) carries the information of HS signal. The summary of PCA is shown in Table 2. While considering the energies of the corresponding $\mathrm{PC}, 1^{\text {st }}$ and $2^{\text {nd }}$ principal components constitute maximum energy which ensures that they had the capability of retrieving the information of primary components as seen in Table 3. So, $1^{\text {st }}$ and $2^{\text {nd }}$ PC featured signals are taken as reference for further processing. 


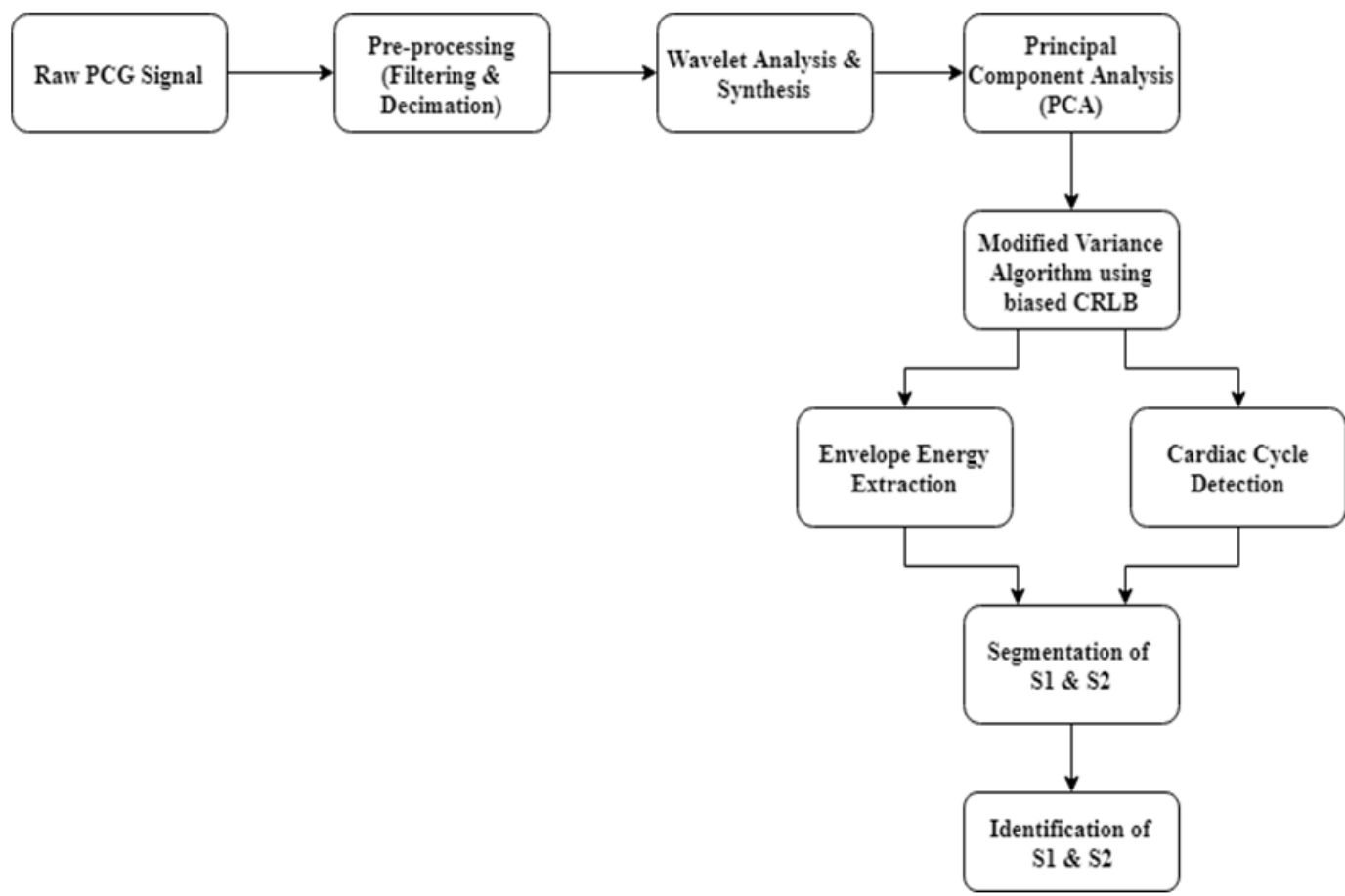

Fig. 1. Block diagram of the HS segmentation process.

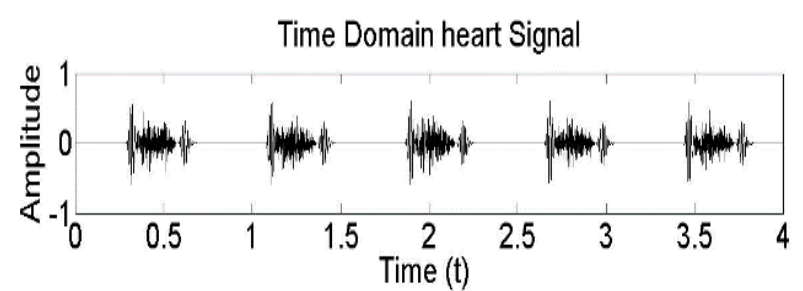

Frequency Spectrum

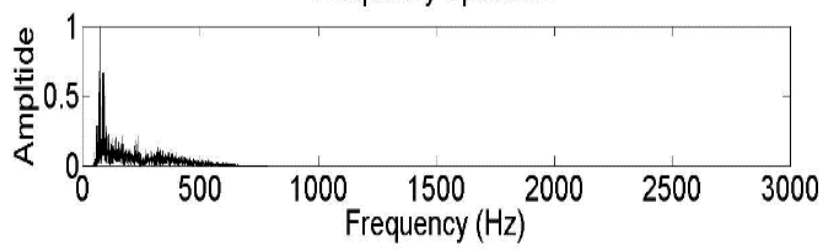

Fig. 2. Aortic stenosis HS signal (top) and its frequency spectrum (bottom)

\section{Cardiac Cycle Detection}

Each PCG signal is a combination of the number of cardiac cycles and splitting them into individual cardiac cycle components is of medical importance. Each cardiac cycle can be obtained by the estimation of start and end positions of the located HSs. This signifies that precise boundaries have to be estimated to obtain accurate cardiac cycles which helps during the segmentation of HSs. Several boundary detection algorithms are proposed by researchers in which, [1] uses different envelope extraction methods to estimate the boundaries of the HS signal and a comparison is made. In [18], a simplicity based heart murmur segmentation is presented which uses mean squared error as a stopping criterion for obtaining the boundaries of S1 and S2, however, the algorithm doesn't work in cases where murmurs overlap $\mathrm{S} 1$ and S2 sounds which is a common issue for PCG signals. Some other researches use subjective knowledge of HS signals like maximum duration of $\mathrm{S} 1$ and $\mathrm{S} 2$, systolic duration and diastolic duration to estimate boundaries that don't work in presence of high noise energies and its performance decreases considerably.

Table II. Summary of levels reflecting S1, S2 and Murmurs using principal component analysis

\begin{tabular}{|c|c|c|c|}
\hline Heart Sounds & $\underline{\text { S1 }}$ & $\underline{\mathbf{S 2}}$ & Murmur components \\
\hline Normal & $1,2,3$ & $1,2,3$ & -- \\
\hline AS & 1,2 & 1,2 & $2,3,4$ \\
\hline AR & 1,2 & 1,2 & $2,3,4$ \\
\hline MS & 1,2 & 1,2 & 2,3 \\
\hline MR & 1,2 & 1,2 & $2,3,4$ \\
\hline PS & 1,2 & 1,2 & $2,3,4$ \\
\hline TS & 1,2 & 1 & $2,3,4$ \\
\hline
\end{tabular}

Table III. Energy distribution of PCG in AS

\begin{tabular}{|c|c|c|c|c|c|}
\hline PCs & PC1 & PC2 & PC3 & PC4 & PC5 \\
\hline $\begin{array}{c}\text { Energy } \\
\%\end{array}$ & $\mathbf{8 2 . 6 4}$ & 16.85 & 0.38 & $\mathbf{0 . 0 9 6}$ & $\mathbf{0 . 0 2 6}$ \\
\hline
\end{tabular}

i. Variance Algorithm for Estimating Cardiac Cycles

A novel variance algorithm is proposed in our study, to estimate the boundary location of the cardiac cycles. The proposed algorithm shown in Fig.3, uses windowed segments to validate the locations of beginning and end position of each cardiac cycle. Variance is calculated for the windowed segments of the first two PC signals, often named as local variances which are compared to the global variances of the original HS signal and the first two PC signals. If the condition is satisfied, it indicates that there is a possibility of the primary HS signal component in that interval. So, to confirm and identify the finer boundary locations, the algorithm is further analyzed by iteratively reducing the width of the window segment until the above-mentioned variance conditions are satisfied. $\sigma_{(.)}$determines the variance of the signal where (.) denotes the corresponding signal parameter. $S_{n}$ determines the $n^{\text {th }}$ sample of the PCG signal having length $N, P_{k}$ determines the $k^{\text {th }}$ sample of the $1^{\text {st }}$ PC of PCA analyzed HS signal having length $K, R_{m}$ determines the $\mathrm{m}^{\text {th }}$ sample of the $2^{\text {nd }} \mathrm{PC}$ of PCA analyzed HS signal having length $\mathrm{M}$. 
This algorithm initially searches for the start position of the $1^{\text {st }}$ cardiac cycle followed by a successive end position which is confirmed by a special condition (1) derived from sampling period $\left(t_{s}\right)$ and maximum possible heart rate $\left(t_{h r}\right)$ for an abnormal patient.

$$
T_{L}=t_{s} * t_{h r}
$$

where, $T_{L}$ determines the minimum time period of the cardiac cycle.

The value of $T_{L}$ varies between individuals, but in general they can be approximated to a constant. For our study, the average heart rate possible for an abnormal condition is considered as 100 beats per minute $[9,16]$. The boundary limit accuracy is confined to a limited number of iterations (itr) which depends on the window size (W) to eliminate false representation. Fig. 4 depicts the normalized mean square error (NMSE) (2) calculated with the reduction of window size iteratively for different cardiac disorders which shows that NMSE deviates from the true value due to smaller window sizes indicating error in capturing boundary locations. To overcome this problem, a threshold (Th) is conditioned which is obtained by using the window size $(\mathrm{W})$

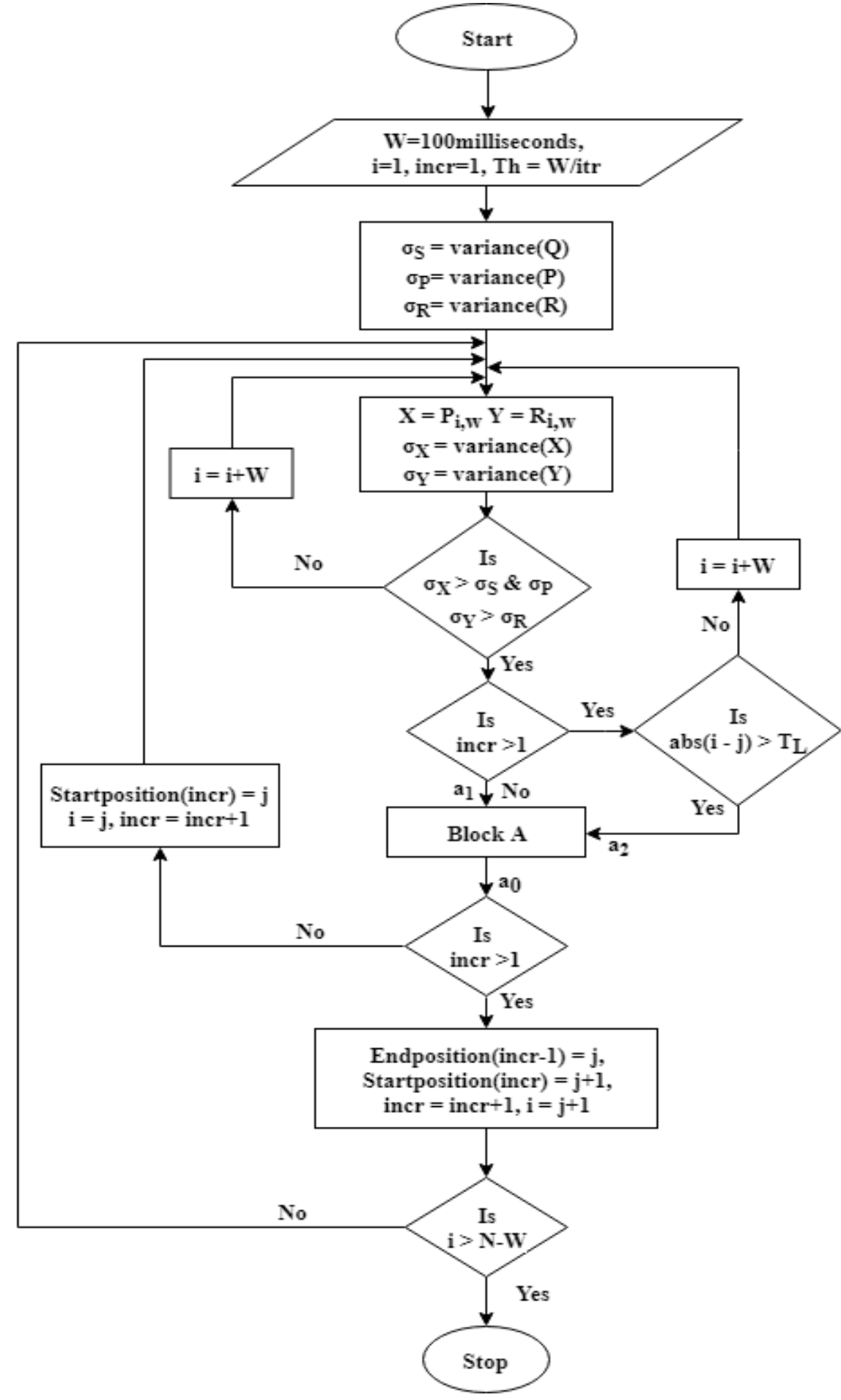

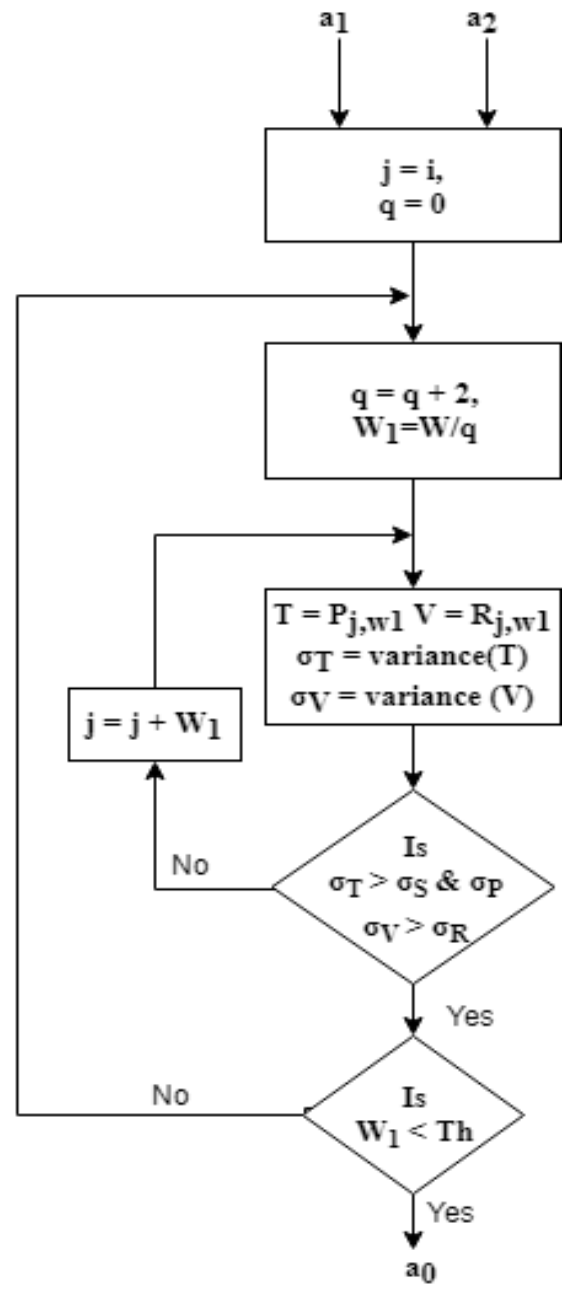

Block A

Fig. 3. Flow diagram to determine beginning and end position of a cardiac cycle. 


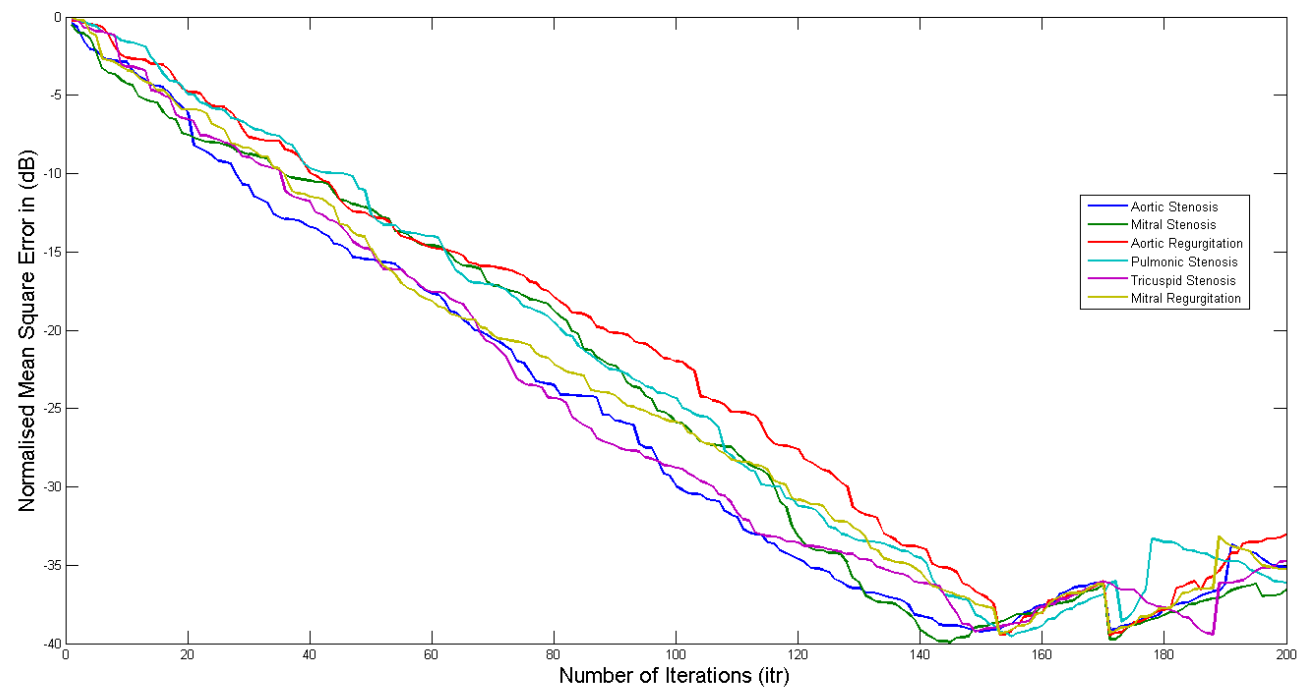

Fig. 4. NMSE showing boundary location accuracy of cardiac cycles using variance algorithm.

Further, the cardiac cycles are detected and the performance of detection is calculated using the knowledge of observed heart cycles $\left(\mathrm{C}_{\mathrm{o}}\right)$ with the actual cycles $\left(\mathrm{C}_{\mathrm{a}}\right)$ marked physically, which can be viewed as the percentage of correctly segmented cardiac cycles $\left(\mathrm{C}_{\text {seg }}\right)$.

$$
C_{\text {seg }}=\frac{C_{o}}{C_{a}} * 100
$$

\section{ii. Modified Variance Algorithm for estimating cardiac cycles}

As the PCG signals are quasi-periodic in nature and the feature extracted PC signals are Gaussian distributed with non-zero mean and variance, estimating HS signal yields significant results. These facts draw the feasibility of applying MVU estimator considering biased CRLB [14] to the HS signals. From the observations of featured PCG signals, one can say that the components exhibit cyclical behavior with a finite number of frequencies. So it is a common practice to determine the presence of strong cyclical components (especially S1 \& S2) by employing Fourier analysis [21, 22]. Considering the signal as Gaussian distributed, the data model can be represented as:

$x[k]=\sum_{q=1}^{M} a_{q} \cos \left(\frac{2 \pi q k}{K}\right)+\sum_{q=1}^{M} b_{q} \sin \left(\frac{2 \pi q k}{K}\right)+w[k]$

Where $k=0,1, \ldots, K-1, w[k]$ is Gaussian noise. The frequencies are assumed to be multiples of the fundamental frequency $f_{l}=1 / K$ as $f_{k}=k / N$. The coefficients $a_{q}$ and $b_{q}$ are to be estimated using biased CRLB.

Let $\mathrm{Y}_{0}$ be the unknown deterministic parameter from a given signal $\mathrm{X}$ having finite frequency range, where $\mathrm{X}$ is characterized by $\mathrm{Y}_{0}$ i.e. $P\left(\mathrm{X} ; \mathrm{Y}_{0}\right)$. Then, the covariance of any biased estimator considering $\hat{Y}$ as an arbitrary estimator of $Y_{0}$ with bias

$$
\mathrm{b}\left(\mathrm{Y}_{0}\right)=E(\hat{\mathrm{Y}})-\mathrm{Y}_{0}
$$

is given by

$$
C_{\hat{\mathrm{Y}}}=E\left\{[\hat{\mathrm{Y}}-E(\hat{\mathrm{Y}})][\hat{\mathrm{Y}}-E(\hat{\mathrm{Y}})]^{*}\right\}
$$

Here, the covariance matrix must satisfy

$$
C_{\hat{\mathrm{Y}}} \geq(I+B) J^{-1}(I+B)^{*}
$$

where, $\mathbf{J}$ and $\mathbf{B}$ are Fisher information matrix and bias gradient matrix which are given by

$$
\begin{aligned}
& J=E\left\{\left[\frac{\partial \log \left(X ; Y_{O}\right)}{\partial Y}\right]^{*}\left[\frac{\partial \log \left(X ; Y_{O}\right)}{\partial Y}\right]\right\} \\
& B=\frac{\partial b\left(Y_{o}\right)}{\partial Y}
\end{aligned}
$$

Since, Fourier analysis confirms orthogonality, to obtain better performance of the estimator we choose to minimize the total variance by considering principal diagonal elements of the covariance matrix which has a constant bias norm.

$$
\operatorname{Tr}\left(C_{\hat{Y}}\right)=\operatorname{Tr}\left[(I+B) J^{-1}(I+B)^{*}\right]
$$

The NMSE calculated by varying the number of coefficients (M) in the Fourier signal indicates that the error reduces significantly with an increase in $\mathrm{M}$ which is shown in table 4. From our analysis, we have chosen $\mathrm{M}=27$ which yields minimum variance with affordable computational complexity. The coefficients obtained constructs a better model with minimum artifacts for the representation of HS signals which are then passed through the proposed variance algorithm (VA) to segment the HS signal into a series of cardiac cycles. The NMSE of the MVA for various disorders with $\mathrm{M}=27$ is shown in Fig. 5 .

\section{iii. Envelope Extraction}

The dynamics of the murmur from the PCG signal can be analyzed only when the primary components (S1 \& S2) are segmented from whole PCG signal. The primary components can be segmented by using the energy and duration of the envelope. Various methods are there for finding the energy and envelope positions in a signal. As primary componentsof HSshave most of the energy concentration at medium levels, Shannon energy (SE) is best suited because of its property of magnifying medium intensity levels while attenuating the low energy noises $[5,25]$.

Table IV. Summary of NMSE in decibels for various $M$ values 


\begin{tabular}{|c|c|c|c|c|c|c|}
\hline $\begin{array}{c}\text { Number of } \\
\text { coefficients }\end{array}$ & \multicolumn{9}{|c|}{ Normalized Mean Square Error (NMSE) in dB } \\
\hline M & AS & MS & AR & PS & TS & MR \\
\hline 7 & -32.26 & -31.87 & -28.88 & -31.35 & -30.96 & -32.17 \\
\hline 11 & -38.55 & -38.28 & -36.84 & -37.83 & -38.17 & -38.45 \\
\hline 15 & -46.15 & -44.9 & -43.56 & -45.5 & -45.83 & -46.7 \\
\hline 19 & -55.63 & -56.28 & -53.25 & -57.14 & -56.46 & -56.95 \\
\hline 23 & -63.26 & -63.8 & -62.34 & -63.58 & -66.05 & -65.82 \\
\hline 27 & -71.35 & -71.03 & $-\mathbf{6 8 . 2 5}$ & -70.82 & -71.23 & -70.76 \\
\hline 35 & -72.68 & -71.78 & -70.62 & -72.84 & -72.47 & -71.85 \\
\hline 43 & -73.83 & -73.46 & -71.85 & -73.27 & -72.63 & -73.05 \\
\hline
\end{tabular}

A 40 millisecond window size is used to calculate Shannon energy with a $50 \%$ overlap. The Shannon energy for the murmur segmented HS signal $P_{t}$ is calculated by:

$$
E=-\frac{1}{N_{s}} \sum_{i=1}^{N_{s}} P_{i}^{2} * \log \left(P_{i}^{2}\right)
$$

where, $N_{s}$ determines windowed samples count.

The energy level positions captured for both HS components and murmurs are recorded for further analysis. Fig. 6 shows the energy that is extracted using Shannon energy for a patient with PS.

\section{Segmentation and Detection of Heart Sounds}

The type of pathology and its auscultation position determines the location and intensity of the murmur in the HS signal [16]. It concludes that the same pathology can have different murmur positions when auscultated at different locations leaving primary components stationary. So instead of murmurs, we segment primary components (S1 \&S2) which have a deterministic pattern. Duringsegmentation, due to valvular dysfunctionality some of the primary HSs may getburied and unidentified. Detecting those peaks can be achieved by considering the difference in the positions of the envelope with the cardiac cycle duration and using automatically reduced thresholding. Along with the stored records, envelope period and consecutive envelop differences are used to derive two thresholds which helps the algorithm in eliminating any false position representation and detect HSs precisely. The thresholds are

$$
\begin{aligned}
& T_{1}=\frac{P_{k}+\left(\left(P_{k+1}-P_{k}\right) / s f_{1}\right.}{T_{q}} \\
& T_{2}=\frac{P_{k}+\left(\left(P_{k+1}-P_{k}\right) / s f_{2}\right.}{T_{q}}
\end{aligned}
$$

where $k=1,2,3, \ldots, L, L=$ total cardiac cycles count, $P_{k}$ denotes the location of featured envelope signal, $T_{q}$ denotes cardiac cycle period at $q=1,2,3, \ldots, L, s f_{1}$, and $s f_{2}$ are scaling factors that change depending on the pathology.

The algorithm has the capability in segmenting even S3 and S4 sounds by considering them as redundant along with the pathological murmurs. The masking of primary components after segmentation is performed using gates as shown in Fig. 7. The obtained gates are overlapped on the PCG signal to extract primary components. Further, identification of S1 and S2 components from segmented HS signals help the researchers to categorize the murmurs based on their occurrence in the cardiac cycle. Along with the subjective systolic and diastolic duration [20], recorded cardiac cycle periods and the calculated envelope extraction positions using (11) from the murmur segmented HS signals are used to identify S1 and S2 components as shown in Fig. 8.

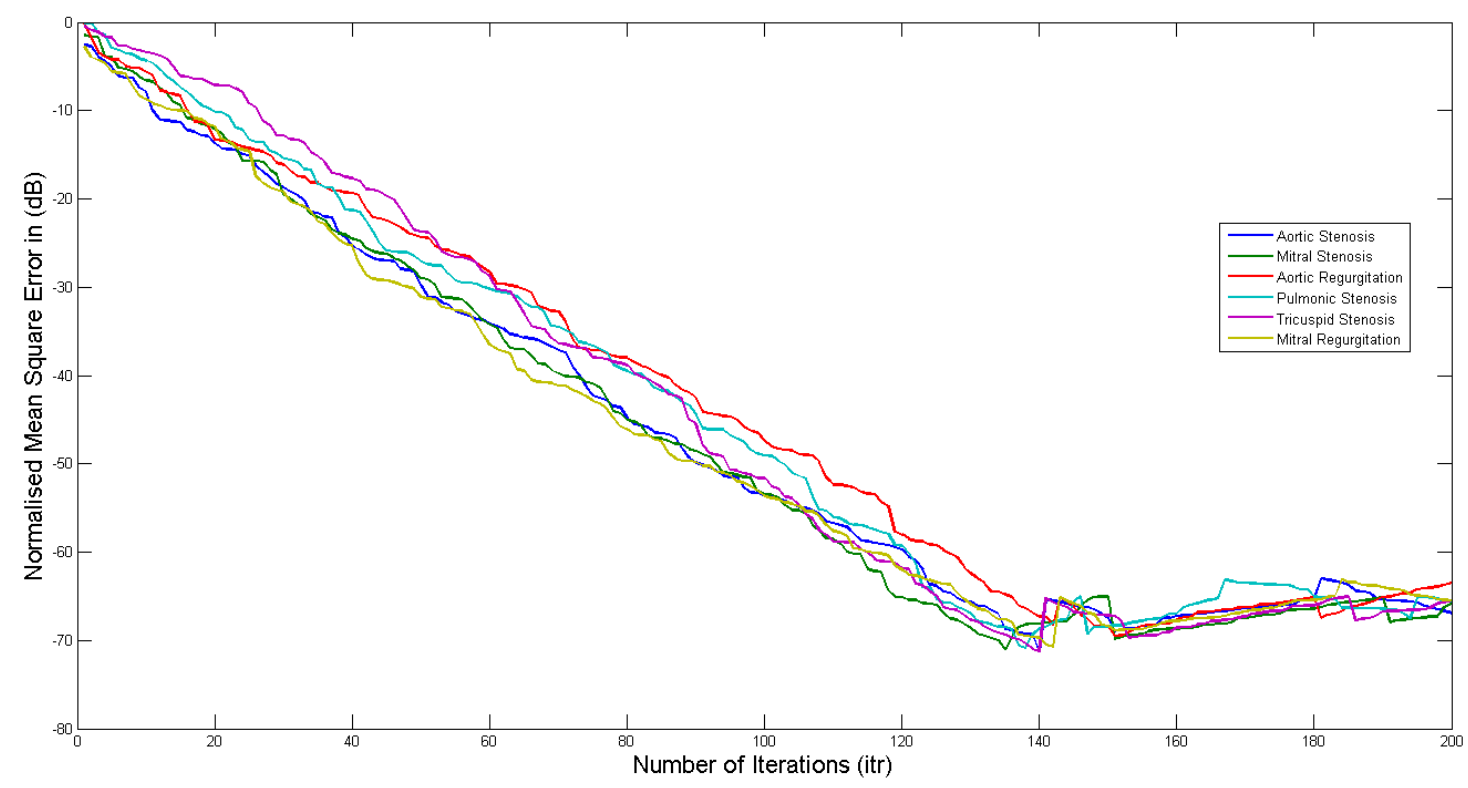

Fig. 5. NMSE showing boundary location accuracy of cardiac cycles using modified variance algorithm with $\mathrm{M=27}$. 

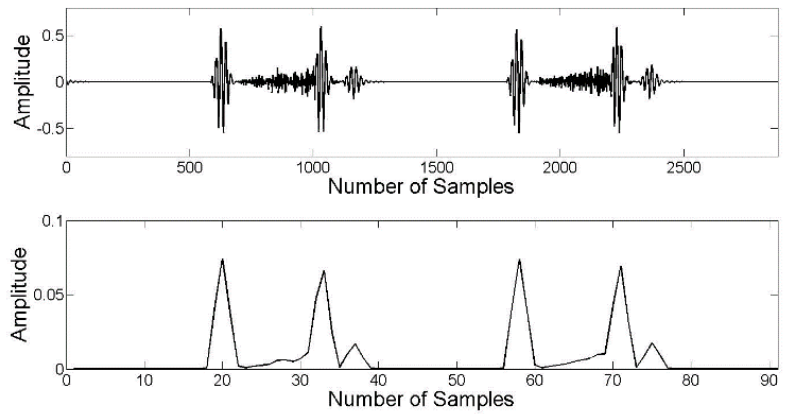

Fig. 6. HS signal of a patient with PS (top) and its energy envelogram (bottom).

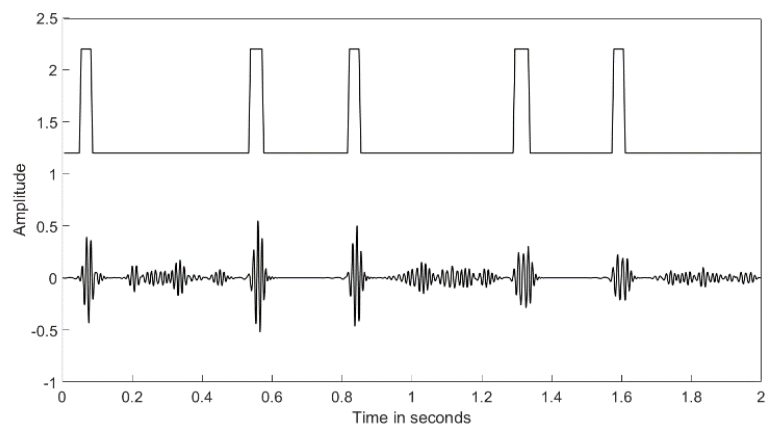

Fig. 7. PCG signal for a patient with MS (bottom) and masking S1 and S2 with gates (top).

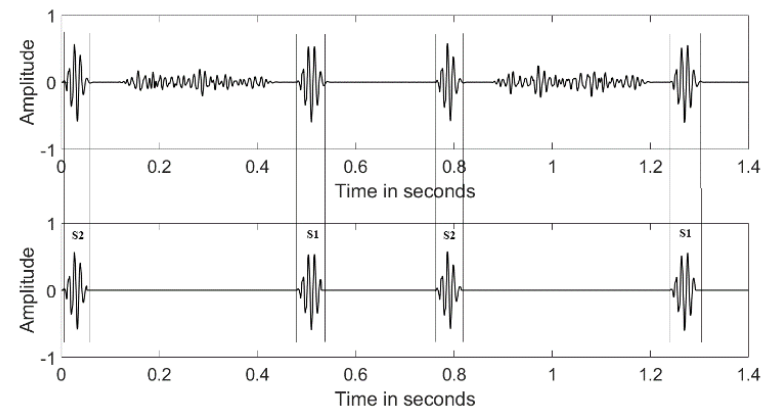

Fig. 8. PCG signal for a patient with MS (top), it's segmented and identified $\mathrm{S} 1, \mathrm{~S} 2$ components (bottom).

\section{DISCUSSION}

The analysis ofHS signals performance is very difficult to achieve. The proposed MVA method exhibits high accuracy levels for both normal and pathological cases. Thepathologies include AS, MS, PS, AR, TS, and MR. The performance is evaluated by using NMSE and correct cardiac cycle segmentation $\left(\mathrm{C}_{\mathrm{seg}}\right)$ which are shown in Tables 5 and 6 . From the tables, it is evident that the MVA method performs efficiently for all set of pathological HSs along with normal HSs. Results show that the MVA scheme performs efficiently for normal subjects with NMSE of $-78.8 \pm 0.15 \mathrm{~dB}$ and segments almost all cardiac cycles correctly with $\mathrm{C}_{\text {seg }}$ of $99.5 \pm 0.5 \%$. Also, significant results are observed for pathological cases with an average NMSE of $70.41 \pm 0.26 \mathrm{~dB}$ and $\mathrm{C}_{\text {seg }}$ of $97.49 \pm 1.03 \%$. The lower NMSE and $\mathrm{C}_{\text {seg }}$ indices for $\mathrm{AR}$ are due to the higher concentration of murmur in the heart sound components. The thresholds $T_{1}$ and $T_{2}$ with varying scale factors help in identifying the systolic and diastolic phases in the heart cycle, thereby identifying S1 and $\mathrm{S} 2$ components clearly.

To examine the efficacy of the proposed MVA scheme, a noise stress test using additive Gaussian noise at various levels of signal to noise ratio (SNR) is performed. As raw PCG signals are prone to noise and thus, are inappropriate for testing under noise stress because the synthetic noise may get merged with the existing noise in the PCG signal, both the featured 1st PC signal (Xf(n)) and murmur segmented HS signal $(\mathrm{Xseg}(\mathrm{n}))$ are used to check the validity of proposed MVA scheme under different noise conditions. Six cardiac cycles are selected each from Xf(n) of Aortic and Pulmonic stenosis cases, and another six cardiac cycles from Xseg(n). The stress test was carried out for six different noise levels $\{0,5,10,20,30,40 \mathrm{~dB}\}$ for both the set of test signals. The evaluation components NMSE and Cseg are calculated for the evaluated test signals and are graphically depicted in fig. 9. From the figure, it is evident that the proposed method performs accurately for the case of Xseg(n) signal. Although $\mathrm{Xf}(\mathrm{n})$ produces compromising result, it is convincing that the method shows an acceptable outcome without much deviation from the existing results (NMSE > $-60 \mathrm{~dB}$ and Cseg> $94 \%$ for $40 \mathrm{~dB}$ SNR). In the case of $\mathrm{Xseg}(\mathrm{n})$, the NMSE crosses -70dB showing approximately 10-4 precision level for the SNR above 40dB and Cseg approaches $100 \%$ accuracy for the SNR above $15 \mathrm{~dB}$. The results obtained from the proposed MVA scheme to various pathological cases and the noise stress test confirms that the method gives prominent results and surpass the ones with its prior VA, PCA and SE methods. However, in some cases specifically AR, the performance of NMSE and Cseg is quite compromising due to the severity of the disease and diagnostic environment, which may allow noise to overlap with the heart sounds.

As heart sounds are categorized based on S1, systolic, S2 and diastolic phases, identifying the position and duration of $\mathrm{S} 1$ and S2 components ascertains the systolic and diastolic periods thereby the murmur location can be identified. Further, the anatomical behavior of the heart states that the diastolic duration is longer than the systolic duration [20]. So, to categorize the cardiac cycle into segments of S1, S2, systole, and diastole, envelope extracted positions of each cardiac cycle, its corresponding cardiac cycle duration and anatomical knowledge are used. The process is conducted for all 4640 correctly identified cardiac cycles and the four phases are identified with an accuracy level of $97.4 \pm 1.2 \%$. The deviation in the accuracy is due to weak S2 sounds that are buried below the threshold level.

\section{CONCLUSION}

The MVA algorithm using biased CRLB is proposed in this study which proved to be a primary tool in segmenting HS signals into cardiac cycles and identifying S1 and S2 components along with systolic and diastolic phases. The cyclo-stationary nature of S1 and S2 components allows theMVA scheme to model the HS signal efficiently with minimum error. The method was conducted on 4746 cardiac cycles which include normal and pathological cases with different severity levels. 
Table V. Summary of normalized mean square error (NMSE) using MVA and other methods.

\begin{tabular}{|c|c|c|c|c|c|c|c|}
\hline \multicolumn{8}{|c|}{$\mathrm{NMSE} \pm$ std (dB) } \\
\hline Subjects & 30 & 11 & 13 & 6 & 7 & 10 & 9 \\
\hline Cardiac Cycles & 1535 & 652 & 782 & 348 & 445 & 498 & 486 \\
\hline PCA & $-31.8 \pm 1.15$ & $-33.46 \pm 1.35$ & $-33.87 \pm 2.16$ & $-28.87 \pm 3.46$ & $-32.95 \pm 2.4$ & $-33.05 \pm 0.53$ & $-32.56 \pm 1.48$ \\
\hline VA & $-42.5 \pm 0.5$ & $-39.23 \pm 0.2$ & $-39.92 \pm 0.08$ & $-36.46 \pm 0.35$ & $-39.33 \pm 0.24$ & $-38.80 \pm 0.15$ & $-37.75 \pm 0.45$ \\
\hline SE & $-32.4 \pm 2.35$ & $-29.86 \pm 1.43$ & $-31.13 \pm 0.56$ & $-27.25 \pm 1.87$ & $-30.76 \pm 2.37$ & $-32.15 \pm 1.62$ & $-30.54 \pm 2.8$ \\
\hline
\end{tabular}

Table VI. Summary of correct cardiac cycle segmentation (Cseg) using MVA and other methods.

\begin{tabular}{|c|c|c|c|c|c|c|c|}
\hline \multicolumn{8}{|c|}{$\mathrm{C}_{\mathrm{seg}} \pm \operatorname{std}(\%)$} \\
\hline Subjects & 30 & 11 & 13 & 6 & 7 & 10 & 9 \\
\hline PCA & $93.5 \pm 1$ & $77.64 \pm 4.82$ & $75.37 \pm 3.57$ & $64.86 \pm 8.55$ & $78.63 \pm 2.47$ & $79.72 \pm 3.86$ & $74.87 \pm 5.76$ \\
\hline VA & $97.5 \pm 0.5$ & $92.56 \pm 2.87$ & $91.82 \pm 1.89$ & $85.43 \pm 3.52$ & $92.67 \pm 2.16$ & $93.38 \pm 1.82$ & $91.76 \pm 3.18$ \\
\hline SE & $92 \pm 2$ & $71.48 \pm 6.73$ & $70.55 \pm 5.8$ & $61.25 \pm 9.47$ & $69.76 \pm 4.92$ & $71.27 \pm 3.82$ & $70.34 \pm 5.93$ \\
\hline
\end{tabular}
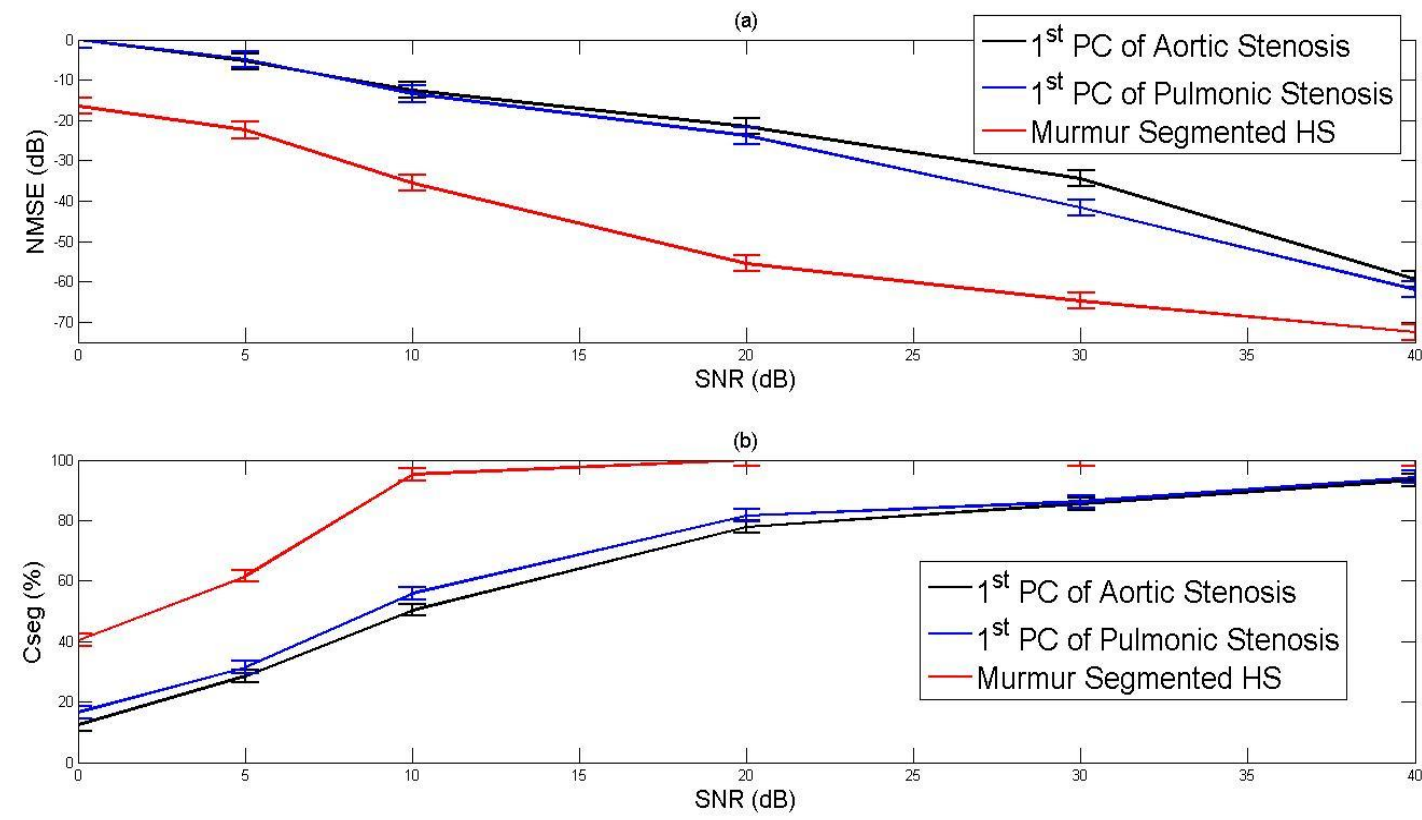

Fig. 9. Mean value with standard deviation of noise stress test (a) NMSE (dB) (b) Cseg (\%). In both cases, featured 1st PC of AS, PS and murmur segmented HS signals are use

Despite of the condition, the proposed scheme shows consistent performance in segmenting and extracting primary components. Further, the noise stress test applied to the segmented heart signals reveal a highly robust response. The computational effort can be reduced further by modeling the HS signal with other nonlinear models that fit better than the Fourier approach. Moreover, an attempt to understand the behavioral pattern of the murmurs can be analyzed from the basis of the proposed scheme and distinguish murmurs based on its structural pattern by using various estimation concepts. Comparison with its prior algorithm (VA) and other works show that the proposed method outperformed in noise minimization, segmentation, and detection. Future work will be mainly focused on modeling the murmur components into structural patterns with their positional responses from PCG signal and design a diagnostic tool that can identify variety of cardiac disorders.

\section{ACKNOWLEDGMENT}

Thanks to GITAM (Deemed to be university) and NIT Rourkela academic staff for their extreme support in providing resources for the project. Besides, we are very much thankful to Dr. Lakshi Prosad Roy for his support in processing and validating data.

\section{REFERENCES}

1. Samjin Choi, Zhongwei Jiang, "Comparison of Envelope Extraction Algorithms for Cardiac Sound Signal Segmentation", Expert Systems with Applications, 2008, Edition 34, Issue 2, pp. 1056-1069.

2. Reed T R, Reed N E and Fritzson P, "Heart sound analysis for symptom detection and computer-aided diagnosis,"Simul. Model. Practice Theory, 12 (2004), pp.129-146. 
3. AbdelghaniDjebbari and FethiBereksiReguig, "Short-Time Fourier Transform Analysis of the Phonocardiogram Signal", $7^{\text {th }}$ IEEE International Conference, 2000, volume 2, pp. 844-847.

4. Jung Jun Lee, Sang Min Lee, In Young Kim, Hong Ki Min, Seung Hong Hong, "Comparison between Short Time Fourier Transform and Wavelet Transform for Feature Extraction of Heart Sound", Proceedings of IEEE Region 10 Conference, TENCON, 1999, volume 2, pp. 1547-1550.

5. H. Liang, S. Lukkarinen, and I. Hartimo, "Heart sound segmentation algorithm based on heart sound envelogram," Computers in Cardiology, volume 24, 1997, pp. 105-108.

6. D. Boutana, M. Benidir, and B. Barkat, "Segmentation and identification of some pathological phonocardiogram signals using time-frequency analysis", IET Signal Processing, 2011, volume 5, pp 527-537.

7. C Ahlstrom, P Hult, P Rask, J.E Karlsson, E Nylander, U Dahlstrom, P Ask, "Feature Extraction for Systolic Heart Murmur Classification", Annals of Biomedical Engineering, November 2006, volume 34, pp. 1666-1677.

8. M.A.R Santos and M. Souza, "Detection of First and Second Cardiac Sounds Based on Time-Frequency Analysis", proceedings $23^{\text {rd }}$ EMBS conference, Istanbul, 2001, pp. 1915-1918.

9. L. Goldberger, David R. Rigney and Bruce J. West, "Chaos and Fractals in Human Physiology," Scientific American, February 1990 pp.421-435.

10. Hong Tang, Ting Li, Yongwan Park, and Tianshuang Qiu. "Separation of Heart Sound Signal from Noise in Joint Cycle Frequency-Time-Frequency Domains Based on Fuzzy Detection." IEEE Transactions on Biomedical Engineering, volume 57, 2010, pp. 2438-2447.

11. Moukadem Ali, Alain Dieterlen, Nicolas Hueber, and Christian Brandt. "A robust heart sounds segmentation module based on S-transform." Biomedical Signal Processing and Control 8, no. 3 2013, pp. 273-281.

12. Chrysa D. Papadaniil and Leontios J.Hadjileontiadis."Efficient Heart Sound Segmentation and Extraction Using Ensemble Empirical Mode Decomposition and Kurtosis Features" IEEE Journal of Biomedical and Health Informatics, volume 18, 2014, pp. 1138-1152.

13. Sunjung Kim and Dosik Hwang. "Murmur-adaptive compression technique for phonocardiogram signals." Electronics Letters, volume 52, 2016, pp. 183-184.

14. Yonina C. Eldar, "Minimum Variance in Biased Estimation: Bounds and Asymptotically Optimal Estimators", IEEE Transactions on Signal Processing, volume 52, 2004, pp. 1915-1930.

15. Loudon, Robert, and Raymond LH Murphy JR. "Lung Sounds'2." Am Rev Respir Dis 130 (1984), pp. 663-673.

16. Paul A, Laizzo (Ed), "Handbook of Cardiac Anatomy, Physiology, and Devices", 2009, $2^{\text {nd }}$ Edition.

17. Stephane Mallat, "A wavelet Tour of Signal Processing, The Sparse Way", An Imprint of Elsevier, 2009, $3^{\text {rd }}$ Edition.

18. D., P. Carvalho,Kumar, M. Antunes, J. Henriques, M. Maldonado, R. Schmidt, and J. Habetha, "Wavelet transform and simplicity based heart murmur segmentation," Proc. Computers in Cardiology, Valencia, pp. 173-176,Spain, 2006

19. Diddi Sandeep Vara Sankar, Lakshi Prosad ARoy, "Principal Component Analysis (PCA) Approach to Segment Primary Components from Pathological Phonocardiogram" IEEE International Conference on Communication and Signal Processing, 2014, pp. 910 914.

20. Hadjileontiadis L.J, Rekanos I.T, "Detection of Explosive Lungs and Bowel Sounds by means of Fractal Dimensions", IEEE Signal Processing Letters, 2003, pp. 311-314.

21. Steven M. Kay, "Fundamentals of Statistical Signal Processing, Estimation Theory", volume 1, 2010.

22. S. F. Yau and Y. Bresler, "A compact Cramer-Rao bound expression for parametric estimation of superimposed signals," IEEE Transactions on Signal Processing, volume 40,1992, pp. 1226-1230

23. Variety of Heart Sound signals taken from Easyauscultation

24. Chengyu Liu et al "An Open Access Database for the Evaluation of Heart Sound Algorithms" Physiological Measurements, volume 37, number 12, 2016

25. Ajith Kumar P C, T V Ananthapadmanabha, "Heart Rate Variability using Shannon Energy", SASTECH, volume 2, November 2006, pp. 23-26.

\section{AUTHORS PROFILE}

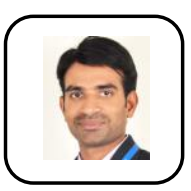

D.Sandeep Vara Sankar received his B.Tech degree from PYDAH affiliated under JNTU-Kakinada and M.Tech degree from NIT Rourkela in the Dept of Electronics and Communications Engineering. Since 2015, He has been working as Assistant Professor at the department of EECE in GITAM University. He published a research book and IEEE paper on cardiovascular disorders. His current research interests includes Bio-medical signal processing, Bio-inspired algorithms and Neural computing.

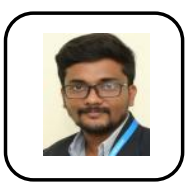

R.Adityareceied his B.Tech degree from ANITS-Andhra University at the department of Electronics and Communication Engineering and M.Tech degree from NIT Rourkela.He has been working as Assistant Professor at the department of EECE. His current research areas include Embedded systems for Automation and controlle design for Robust stabilization of uncertain systems.

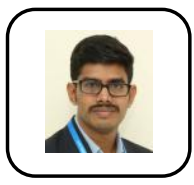

S Gopi Krishna received his B.Tech degree from Vignan affiliated under JNTUK at the department of Electronics and Communication Engineering and M.Tech degree from NIT Surat. He is currently working as Assistant Professor in GITAM University. His research interests are Signal processing and Communication system design.

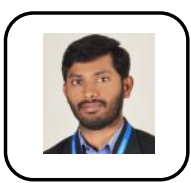

P Atchuta Rao received his B.Tech degree from Koushik college affiliated under JNTUK at the department of Electronics and Communication Engineering and M.Tech degree from NIT Rourkela. He is currently working as Assistant Professor in GITAM University. His research interests are Antenna design and signal analysis. 